\title{
Glueball Properties at Finite Temperature
}

\author{
Noriyoshi Ishii $^{\mathrm{a} *}$, Hideo Suganuma ${ }^{\mathrm{b}}$, Hideo Matsufuru ${ }^{\mathrm{c}}$ \\ a Radiation Laboratory, The Institute of Physical and Chemical Research (RIKEN), \\ 2-1 Hirosawa, Wako, Saitama 351-0198, JAPAN \\ b Tokyo Institute of Technology, \\ 2-12-1 Ohkayama, Meguro, Tokyo 152-8552, JAPAN \\ ${ }^{c}$ Yukawa Institute for Theoretical Physics, Kyoto University, \\ Kitashirakawa-Oiwake, Sakyo, Kyoto 606-8502, JAPAN
}

We study the glueball properties at finite temperature below $T_{c}$ using $\mathrm{SU}(3)$ anisotropic quenched lattice QCD with $\beta=6.25$, the renormalized anisotropy $\gamma=a_{s} / a_{t}=4$ and $20^{3} \times N_{t}\left(N_{t}=35,36,37,38,40,43,45,50,72\right)$. From the temporal correlation analysis with the smearing method, we observe the mass reduction of about $20 \%$ for the lowest $0^{++}$glueball as $m_{\mathrm{G}}(T)=1.25 \pm 0.1 \mathrm{GeV}$ for $0.8 T_{c}<T<T_{c}$ in comparison with $m_{\mathrm{G}} \simeq 1.5 \sim 1.7$ $\mathrm{GeV}$ at $T \sim 0$.

\section{INTRODUCTION}

Finite temperature QCD, including the quark gluon plasma(QGP) physics, is one of the most interesting subjects. Even in the hadronic phase, a lot of effective models suggest the changes in the hadronic properties. However, lattice QCD studies of thermal hadron properties have been inadequate until quite recent. This is due to the difficulty in measuring hadronic two-point correlators at finite temperature. At high temperature, due to the shrink in the temporal extention and the consequent decrease in the number of data, the mass measurement from the temporal correlations is difficult. Hence, finite temperature mass shifts have been studied through the spatial correlations, which, however, is afflicted with the mixture of large Matsubara frequencies 11. Recently, the use of anisotropic lattice is established [2], which has the finer temporal lattice spacing $a_{t}$ than the spatial one $a_{s}$. In this way, available data increases, and the accurate mass measurement directly from the temporal correlations becomes possible [3 5]. Here, we report the thermal properties of the $0^{++}$glueball based on the quenched $\mathrm{SU}(3)$ anisotropic lattice QCD.

\footnotetext{
*The lattice calculations have been performed on NEC-
} SX5 at Osaka University.

\section{SMEARING METHOD}

We consider the glueball correlator as $G(t)=$ $\langle O(t) O(0)\rangle, O(t)=\sum_{\vec{x}}(\tilde{O}(\vec{x}, t)-\langle\tilde{O}\rangle)$ where $\tilde{O}(\vec{x}, t)=\operatorname{Re} \operatorname{Tr}\left(P_{12}(\vec{x}, t)+P_{23}(\vec{x}, t)+P_{31}(\vec{x}, t)\right)$ denotes an interpolating field for the $0^{++}$glueball. $P_{i j}(\vec{x}, t) \in \mathrm{SU}(3)$ denotes the plaquette operator in the $i$ - $j$-plain. We apply the spectral representation to $G(t)$ to have $G(t) / G(0)=\sum C_{n} e^{-E_{n} t}$, where $C_{n}=|\langle n|O| 0\rangle|^{2} / \sum|\langle k|O| 0\rangle|^{2}$ with $E_{n}$ being the energy of the $n$-th excited state $|n\rangle$. Here, $|0\rangle$ denotes the vacuum and $|1\rangle$ denotes the ground state glueball. $C_{n}$ is non-negative with $\sum C_{n}=1$. It is known that $G(t)$ receives large contributions from the excited states and the ground state contribution is small. As a consequence, the extracted mass always behaves much heaver than the ground-state mass. The problem originates from the fact that the "size" of the ordinary plaquette operator is $O\left(a_{s}\right)$, which is much smaller than the physical size $R$ of the glueball. Hence, the problem can be resolved by generating physically extended glueball operators, which is achieved by the smearing method [6]. This extended operator is refered to as the smeared operator, which is obtained by replacing the ordinary link variables 
$U_{i}(s)$ in the plaquette operator $P_{i j}(s)$ by the fat link variables $\bar{U}_{i}(s) \in \mathrm{SU}(3) . \quad \bar{U}_{i}(s)$ is defined so as to maximize $\operatorname{Re} \operatorname{Tr}\left(\bar{U}_{i}^{\dagger}(s) V_{i}(s)\right)$, where $V_{i}(s) \equiv \alpha U_{i}(s)+\sum_{j \neq i, \pm} U_{ \pm j}(s) U_{i}(s \pm \hat{j}) U_{ \pm j}^{\dagger}(s+\hat{i})$ with $U_{-\mu}(s) \equiv U_{\mu}^{\dagger}(s-\hat{\mu})$. $\alpha$ is a real parameter. The summation involves only the spatial directions to avoid the temporal nonlocality. Note that $\bar{U}_{i}(s)$ holds the same gauge transformation properties as $U_{i}(s)$. We refer to the fat link defined in this way as the first fat link $U_{i}^{(1)}(s)$. The $n$-th fat link $U_{i}^{(n)}(s)$ is defined iteratively by $U_{i}^{(n)}(s) \equiv$ $\bar{U}_{i}^{(n-1)}(s)$ starting from $U_{i}^{(1)}(s) \equiv \bar{U}_{i}(s)$. The plaquette operator constructed with $U_{i}^{(n)}(s)$ is refered to as the $n$-th smeared operator. We next consider the size of the $n$-th smeared operator. By using the linearization and the continuum approximation, we obtain the diffution equation as

$\frac{\partial}{\partial n} \phi_{i}(n ; \vec{x})=D \triangle \phi_{i}(n ; \vec{x}), \quad D \equiv \frac{a_{s}^{2}}{\alpha+4}$.

$\phi_{i}(n ; \vec{x})$ describes the distribution of the gluon field $A_{i}(\vec{x}, t)$ in the $n$-th smeared plaquette. In the $n$-th smeared plaquette located at the origin $\vec{x}=\overrightarrow{0}$, the gauge field $A_{i}(\vec{x}, t)$ is distributed in the Gaussian form as $e^{-\vec{x}^{2} /(4 D n)} /(4 \pi D n)^{3 / 2}$. Hence the size of the operator is estimated as

$R \equiv \sqrt{\left\langle\vec{x}^{2}\right\rangle}=\sqrt{6 D n}=a_{s} \sqrt{\frac{6 n}{\alpha+4}}$.

The original aim of the smearing method is the accurate mass measurement by maximizing the ground-state overlap. However, it can be also used to give a rough estimate of the physical glueball size. In fact, once the maximum overlap is achieved with some $n$ and $\alpha$, the glueball size can be estimated with Eq.(2).

\section{SU(3) LATTICE QCD RESULT}

We use the $\mathrm{SU}(3)$ anisotropic lattice plaquette action as $S_{G}=\frac{\beta}{N_{c}} \frac{1}{\xi_{0}} \sum_{s, i<j \leq 3} \operatorname{Re} \operatorname{Tr}\left(1-P_{i j}(s)\right)+$ $\frac{\beta}{N_{c}} \xi_{0} \sum_{s, i \leq 3} \operatorname{Re} \operatorname{Tr}\left(1-P_{i 4}(s)\right)$ with the plaquette operator $P_{\mu \nu}(s)$ in the $\mu$ - $\nu$-plane. The lattice pa-

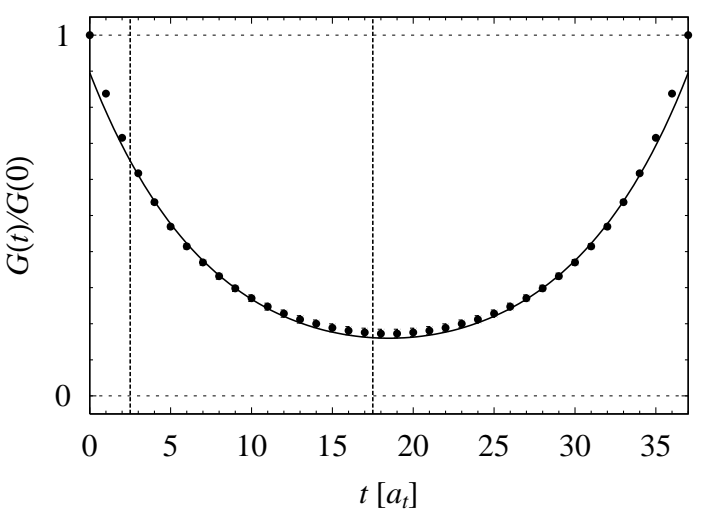

Figure 1. The $0^{++}$glueball correlator $G(t) / G(0)$ for $N_{\text {smear }}=40$ at $T=245 \mathrm{MeV}\left(N_{t}=37\right)$. The statistical errors are hidden within the symbols. The solid line denotes the best single hyperbolic cosine fit performed in the interval indicated by vertical dotted lines.

rameter and the bare anisotropy parameter are fixed as $\beta=6.25, \xi_{0}=3.2552$, respectively. These parameters reproduce the spatial lattice spacing as $a_{s}^{-1}=2.272(16) \mathrm{GeV}$, and the temporal one as $a_{t}^{-1}=9.088(64) \mathrm{GeV}$. The renormalized anisotropy is $\gamma \equiv a_{s} / a_{t}=4$ [2]. Here, the scale unit is determined by reproducing the string tension as $\sqrt{\sigma}=427 \mathrm{MeV}$ from the onaxis data of the static inter-quark potential. The pseudo-heat-bath algorithm is used to update the gauge field configurations on the lattice of the sizes $20^{3} \times N_{t}$ with various $N_{t}$ listed in Table 1. For each temperature, we pick up gauge field configurations every 100 sweeps for measurements, after skipping more than 20,000 sweeps of the thermalization. The numbers of gauge configurations used in our calculations are summarized in Table 1. From the analysis of the Polyakov loop, we find $T_{c} \simeq 260 \mathrm{MeV}$. To enhance the ground-state contribution, we adopt the smearing method with $\alpha=2.1$.

In Fig.11, the $0^{++}$glueball correlator $G(t) / G(0)$ for $N_{\text {smear }}=40$ at $T=245 \mathrm{MeV}$ is shown. The statistical errors are estimated with the jack- 
Table 1

The lattice QCD result for the lowest $0^{++}$glueball mass at finite temperature. The temporal lattice points $N_{t}$, the corresponding temperature $T$, the lowest $0^{++}$glueball mass $m_{\mathrm{G}}(T)$, the maximal value of the ground-state overlap $C^{\text {max }}$, uncorrelated $\chi^{2} / N_{\mathrm{DF}}$, the best smearing number $N_{\text {smear }}^{\text {best }}$, the smearing window $N_{\text {smear }}^{\text {window }}$, the number of gauge configurations $N_{\text {conf }}$ and the glueball size $R \simeq\left\langle\sqrt{\vec{x}^{2}}\right\rangle$ are listed.

\begin{tabular}{ccccccccc}
\hline$N_{t}$ & $T[\mathrm{MeV}]$ & $m_{G}[\mathrm{GeV}]$ & $C^{\text {max }}$ & $\chi^{2} / N_{\text {DF }}$ & $N_{\text {smear }}^{\text {best }}$ & $N_{\text {smear }}^{\text {window }}$ & $N_{\text {conf }}$ & $R[\mathrm{fm}]$ \\
\hline 72 & 125 & $1.54(1)$ & $0.981(6)$ & 0.23 & 41 & $32 \sim 51$ & 1284 & $0.49 \sim 0.61$ \\
50 & 182 & $1.43(2)$ & $0.967(8)$ & 0.02 & 45 & $36 \sim 55$ & 1051 & $0.52 \sim 0.64$ \\
45 & 202 & $1.48(2)$ & $0.971(8)$ & 0.08 & 37 & $30 \sim 46$ & 1382 & $0.47 \sim 0.58$ \\
43 & 210 & $1.30(2)$ & $0.945(8)$ & 0.35 & 40 & $32 \sim 52$ & 1197 & $0.49 \sim 0.62$ \\
40 & 227 & $1.29(2)$ & $0.935(6)$ & 1.22 & 40 & $32 \sim 51$ & 2021 & $0.49 \sim 0.61$ \\
38 & 239 & $1.36(2)$ & $0.935(7)$ & 1.00 & 38 & $30 \sim 50$ & 2030 & $0.47 \sim 0.61$ \\
37 & 245 & $1.18(3)$ & $0.889(12)$ & 0.91 & 43 & $35 \sim 53$ & 2150 & $0.51 \sim 0.63$ \\
36 & 252 & $1.38(2)$ & $0.948(8)$ & 0.09 & 36 & $29 \sim 46$ & 1744 & $0.46 \sim 0.58$ \\
35 & 259 & $1.26(2)$ & $0.931(7)$ & 1.15 & 40 & $31 \sim 51$ & 2244 & $0.48 \sim 0.61$ \\
\hline
\end{tabular}

knife analysis. The solid line denotes the best single hyperbolic cosine fitting as $G(t) / G(0)=$ $C\left(e^{-m_{\mathrm{G}} t a_{t}}+e^{-m_{\mathrm{G}}\left(N_{t}-t\right) a_{t}}\right)$, performed in the interval indicated by vertical dotted lines. In the most suitable smearing number $N_{\text {smear }}^{\text {best }}$, the ground-state overlap $C$ is maximized and the mass $m_{G}$ is minimized, which indicates the achievement of the ground-state enhancement. In practical calculations, the maximum overlap and the mass minimization are achieved at almost the same $N_{\text {smear }}$, and both of these two conditions would work as an indication of the maximal ground-state enhancement. Here, we take the maximum ground-state overlap condition.

In Table 1, we summarize the $\mathrm{SU}(3)$ lattice QCD result of the lowest $0^{++}$glueball mass $m_{\mathrm{G}}(T)$ at various temperatures. We observe that about $20 \%$ mass reduction of the lowest $0^{++}$ glueball near $T_{c}$ as $m_{\mathrm{G}}(T)=1.25 \pm 0.1 \mathrm{GeV}$ for $0.8 T_{c} \leq T \leq T_{c}$ in comparison with $m_{\mathrm{G}}(T \sim 0) \simeq$ $1.5 \sim 1.7 \mathrm{GeV}[7,8]$. To estimate the glueball size, we search $N_{\text {smear }}^{\text {best }}$ which achieves the maximum overlap as $C=C^{\max }$. Considering about 0.5 $\%$ errors of $C$, we define the "smearing window" $N_{\text {smear }}^{\text {window }}$ where $C \geq 0.995 C^{\text {max }}$ is satisfied. From Eq.(2) with $N_{\text {smear }}^{\text {window }}$, we estimate the glueball size as $R \simeq 0.5 \sim 0.6 \mathrm{fm}$ both at low and high temperatures. In Table 1, we summarize the lowest $0^{++}$ glueball mass $m_{\mathrm{G}}(T)$, the ground-state overlap $C^{\text {max }}$, uncorrelated $\chi^{2} / N_{\mathrm{DF}}, N_{\text {smear }}^{\text {best }}, N_{\text {smear }}^{\text {window }}$, the number of gauge configurations $N_{\text {conf }}$ and the glueball size $R$.

\section{SUMMARY}

We have studied the $0^{++}$glueball properties at finite temperature using $\mathrm{SU}(3)$ anisotropic quenched lattice QCD with more than 1,000 gauge configurations at each temperature. From the temporal correlation analysis with the smearing method, we have observed about $20 \%$ mass reduction of the lowest $0^{++}$glueball as $m_{G}(T)=$ $1.25 \pm 0.1 \mathrm{GeV}$ for $0.8 T_{c}<T<T_{c}$, while no significant change has been observed for the glueball size as $R \simeq 0.5 \sim 0.6 \mathrm{fm}$.

\section{REFERENCES}

1. C. DeTar and J. B. Kogut, Phys. Rev. Lett. 59 (1987) 399.

2. T. R. Klassen, Nucl. Phys. B533 (1998) 557.

3. QCD-Taro Collaboration, Phys. Rev. D63 (2001) 054501.

4. N. Ishii, H. Suganuma and H. Matsufuru, Proc. of Lepton Scattering, Hadrons and QCD, (World Scientific, 2001).

5. N. Ishii, H. Suganuma and H. Matsufuru, hep-lat/0109011

6. APE Collaboration,Phys.Lett.B192(1987)163.

7. C. J. Morningstar and M. Peardon, Phys. Rev. D60 (1999) 034509.

8. J. Sexton, A.Vaccarino and D. Weingarten, Phys. Rev. Lett. 75 (1995) 4563. 